\title{
Research on Mathematical Model of Anti-twist Braided Wire Rope
}

\author{
Chao $\mathrm{Fu}^{1, \mathrm{a}}$, Hui Wang ${ }^{1, \mathrm{~b}}$, Xia Zhao ${ }^{1, \mathrm{c}}$, Lingdong Sun ${ }^{1, \mathrm{~d}}$ and Ping Zhao ${ }^{1, \mathrm{e}}$ \\ ${ }^{1}$ School of Mechanical Engineering, University of Jinan, Jinan 250022, China. \\ afc15275168268@163.com, ${ }^{b}$ me_wangh@ujn.edu.cn, cjz1015zhaoxia@163.com, ${ }^{\mathrm{d}} 15275160225$ \\ @126.com, ${ }^{\mathrm{e} z h p 1763320464 @ 163 . c o m}$
}

Keywords: anti-twist wire rope; mathematical model; differential geometry.

\begin{abstract}
The mathematical model of anti-twist braided wire rope structure is the basis for studying the mechanical problems of wire rope. According to the forming principle of anti-twist wire rope, the structure and dimensional characteristics of wire rope are analyzed. Applying the differential geometry theory and principle of coordinate transformation, the spatial curve parametric equations were derived. Deduced the mathematical model of anti-twist braided wire rope. On the basis of this mathematical model ,a YS11-8×19 wire rope was taken as an example based on the spatial curve parametric equations, an geometric entity model of the anti-twist braided wire rope was established using the three-dimension software. Compared geometric model with actual anti-twist braided wire rope, errors of structure parameters are in the range of allowable error. The mathematic model's veracity is verified, which lay the foundation for finite element analysis of wire rope.
\end{abstract}

\section{Introduction}

Generally speaking, wire rope can be classified into two categories: lay wire ropes and braided wire ropes Braided wire ropes were woven by a group of anti-twist rope to twist left and right cross to a group of single-strand twisted rope. Due to a non-rotating feature, braided wire ropes are widely used in tension stringing transmission line as a guide rope and leashes. Therefore, the safety of braided wire ropes are highly valued.

Scholars at home and abroad did a more in-depth study on ordinary rope twisting class modeling and mechanical analysis. ${ }^{[1-6]}$ Information retrieval at home and abroad have not yet found the research of anti-twist braided wire rope. Therefore, this paper studied the mathematical model for anti-twisting braided wire rope, established the geometric model of anti-twist braided wire rope. The study will provide strong support to mechanics, wire rope design, manufacture and tensile testing theory and technology, and has important application value.

\section{Modeling Theory Geometric model}

Coordinate Transformation of Wire Rope. Braided wire ropes exist rotation and revolution of wires and strands during the forming process, which relating to the point of rotation around any axis space. This transformation process is more complicated. As shown in Figure 1, Set point $P(x, y, z)$ rotation $\theta$ revolve axis $P_{1} P_{2}$. Seven basic transformations as follows.

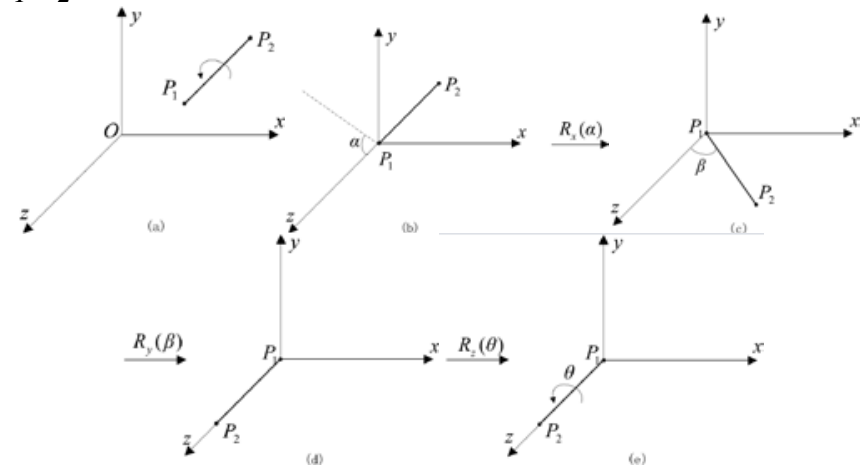

Fig. 1 Revolve any axis $P_{1} P_{2}$ rotation step schematic diagram 
(1) $T\left(-x_{1},-y_{1},-z_{1}\right)$, Make point $P_{1}$ coincides with the origin(Fig.1(b));

(2) $R_{x}(a)$, Make $P_{1} P_{2}$ fall into the plane $x O y$ (Fig.c));

(3) $R_{y}(\beta)$, Make $P_{1} P_{2}$ coincide with the axis $z$ (Fig. (d));

(4) $R_{z}(\theta)$, Rotation $\theta$ revolve axis $P_{1} P_{2}$. (Fig. (e));

(5) $R_{y}(-\beta)$, Make inverse transformation for the transformation 3;

(6) $R_{x}(-a)$, Make inverse transformation for the transformation 2;

(7) $T\left(x_{1}, y_{1}, z_{1}\right)$, Make inverse transformation for the transformation 1.

Forming Principle of Braided Wire Rope. According to the strand cross-sectional position distribution of anti-twist braided wire rope, Combined with the weaving of actual production , This article assumes that anti-twisting steel cord to weave braid forming process can be seen as the point $M$ on the one hand shares in a plane around the oval uniform rotation, on the other hand along a straight line perpendicular to the plane do uniform motion. The forming principle shown in Fig. 2.

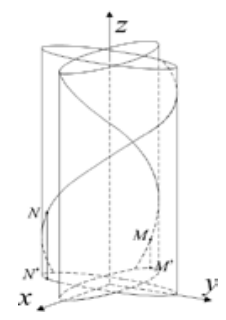

Fig. 2 Strands woven forming schematic diagram

Strands centerline expression as formula (1):

$$
R=\left\{\begin{array}{l}
x_{A} \\
y_{A} \\
z_{A}
\end{array}\right\}=\left\{\begin{array}{l}
a \cos \theta \\
b \sin \theta \\
c \theta
\end{array}\right\}
$$

In the formula, $a$ represent ellipse semimajor, $b$ represent ellipse semiminor, $c=$ Pitch $/ 2 \pi, \theta$ represent elliptic centrifugal.

Mathematical Model of Anti-twist Braided Wire Rope.Shares of a space curve to the right to braid rope molding, $R$ can be expressed as the formula (2):

$R_{\text {Right-handed }}=\left\{\begin{array}{l}x_{A} \\ y_{A} \\ z_{A}\end{array}\right\}=\left\{\begin{array}{l}a \cos \left(\theta+\theta_{0}\right) \\ b \sin \left(\theta+\theta_{0}\right) \\ c \theta\end{array}\right\}$

Lateral wire wire-handed manner in stocks is wound with $Q$ in the Frenet frame $t-n-b$ expression for the formula (3):

$Q_{\text {Right-handed }}^{\prime}=\left\{\begin{array}{l}r_{s} \cos \left(\theta_{s}+\theta_{s 0}\right) \\ r_{s} \sin \left(\theta_{s}+\theta_{s 0}\right) \\ 0\end{array}\right\}$

Shares in the ropes with a right to the conversion matrix $T$ handed knitting formed expression of formula (4):

$$
\begin{aligned}
& T=\left[\begin{array}{cl}
\frac{-a \cos \left(\theta+\theta_{0}\right)}{\sqrt{a^{2} \cos ^{2}\left(\theta+\theta_{0}\right)+b^{2} \sin ^{2}\left(\theta+\theta_{0}\right)}} & \frac{b c \sin \left(\theta+\theta_{0}\right)}{\sqrt{a^{2} \cos ^{2}\left(\theta+\theta_{0}\right)+b^{2} \sin ^{2}\left(\theta+\theta_{0}\right)} \sqrt{a^{2} \sin ^{2}\left(\theta+\theta_{0}\right)+b^{2} \cos ^{2}\left(\theta+\theta_{0}\right)+c^{2}}} \\
\frac{-b \sin \left(\theta+\theta_{0}\right)}{\sqrt{a^{2} \cos ^{2}\left(\theta+\theta_{0}\right)+b^{2} \sin ^{2}\left(\theta+\theta_{0}\right)}} & \frac{-a c \cos \left(\theta+\theta_{0}\right)}{\sqrt{a^{2} \cos ^{2}\left(\theta+\theta_{0}\right)+b^{2} \sin ^{2}\left(\theta+\theta_{0}\right)} \sqrt{a^{2} \sin ^{2}\left(\theta+\theta_{0}\right)+b^{2} \cos ^{2}\left(\theta+\theta_{0}\right)+c^{2}}} \\
0 & \frac{a b}{\sqrt{a^{2} \cos ^{2}\left(\theta+\theta_{0}\right)+b^{2} \sin ^{2}\left(\theta+\theta_{0}\right)} \sqrt{a^{2} \sin ^{2}\left(\theta+\theta_{0}\right)+b^{2} \cos ^{2}\left(\theta+\theta_{0}\right)+c^{2}}}
\end{array}\right. \\
& \left.\begin{array}{c}
\frac{-a \sin \left(\theta+\theta_{0}\right)}{\sqrt{a^{2} \sin ^{2}\left(\theta+\theta_{0}\right)+b^{2} \cos ^{2}\left(\theta+\theta_{0}\right)+c^{2}}} \\
\frac{b \cos \left(\theta+\theta_{0}\right)}{\sqrt{a^{2} \sin ^{2}\left(\theta+\theta_{0}\right)+b^{2} \cos ^{2}\left(\theta+\theta_{0}\right)+c^{2}}} \\
\frac{c}{\sqrt{a^{2} \sin ^{2}\left(\theta+\theta_{0}\right)+b^{2} \cos ^{2}\left(\theta+\theta_{0}\right)+c^{2}}}
\end{array}\right]
\end{aligned}
$$

According to the spatial coordinate transformation principle, $Q$ in the global coordinate system $X Y Z$ expression in the formula (5): 


$$
Q=\left[\begin{array}{cc}
\frac{-r_{s} a \cos \left(\theta+\theta_{0}\right) \cos \left(\theta_{s}+\theta_{s o}\right)}{\sqrt{a^{2} \cos ^{2}\left(\theta+\theta_{0}\right)+b^{2} \sin ^{2}\left(\theta+\theta_{0}\right)}}+\frac{b c r_{s} \sin \left(\theta+\theta_{0}\right) \sin \left(\theta_{s}+\theta_{s o}\right)}{\sqrt{a^{2} \cos ^{2}\left(\theta+\theta_{0}\right)+b^{2} \sin ^{2}\left(\theta+\theta_{0}\right)} \sqrt{a^{2} \sin ^{2}\left(\theta+\theta_{0}\right)+b^{2} \cos ^{2}\left(\theta+\theta_{0}\right)+c^{2}}} \\
\frac{-r_{s} b \sin \left(\theta+\theta_{0}\right) \cos \left(\theta_{s}+\theta_{s o}\right)}{\sqrt{a^{2} \cos ^{2}\left(\theta+\theta_{0}\right)+b^{2} \sin ^{2}\left(\theta+\theta_{0}\right)}}-\frac{a r_{s} \cos \left(\theta+\theta_{0}\right) \sin \left(\theta_{s}+\theta_{s o}\right)}{\sqrt{a^{2} \cos ^{2}\left(\theta+\theta_{0}\right)+b^{2} \sin ^{2}\left(\theta+\theta_{0}\right)} \sqrt{a^{2} \sin ^{2}\left(\theta+\theta_{0}\right)+b^{2} \cos ^{2}\left(\theta+\theta_{0}\right)+c^{2}}} \\
\frac{a b r_{s} \sin \left(\theta_{s}+\theta_{s o}\right)}{\sqrt{a^{2} \cos ^{2}\left(\theta+\theta_{0}\right)+b^{2} \sin ^{2}\left(\theta+\theta_{0}\right)} \sqrt{a^{2} \sin ^{2}\left(\theta+\theta_{0}\right)+b^{2} \cos ^{2}\left(\theta+\theta_{0}\right)+c^{2}}}
\end{array}\right]
$$

For anti-twisting braided wire rope, the strands to weave the right, the right to the wire wound steel siding space vector expression is quadratic equation (6):

$$
P=\left[\begin{array}{c}
\frac{-r_{s} a \cos \left(\theta+\theta_{0}\right) \cos \left(\theta_{s}+\theta_{s o}\right)}{\sqrt{a^{2} \cos ^{2}\left(\theta+\theta_{0}\right)+b^{2} \sin ^{2}\left(\theta+\theta_{0}\right)}}+\frac{b c r_{s} \sin \left(\theta+\theta_{0}\right) \sin \left(\theta_{s}+\theta_{s o}\right)}{\sqrt{a^{2} \cos ^{2}\left(\theta+\theta_{0}\right)+b^{2} \sin ^{2}\left(\theta+\theta_{0}\right)} \sqrt{a^{2} \sin ^{2}\left(\theta+\theta_{0}\right)+b^{2} \cos ^{2}\left(\theta+\theta_{0}\right)+c^{2}}}+a \cos \left(\theta+\theta_{0}\right) \\
\frac{a c r_{s} \cos \left(\theta+\theta_{0}\right) \sin \left(\theta_{s}+\theta_{s o}\right)}{\sqrt{a^{2} \cos ^{2}\left(\theta+\theta_{0}\right)+b^{2} \sin ^{2}\left(\theta+\theta_{0}\right)}}-\frac{\left.\theta_{s}\right)}{\sqrt{a^{2} \cos ^{2}\left(\theta+\theta_{0}\right)+b^{2} \sin ^{2}\left(\theta+\theta_{0}\right)} \sqrt{a^{2} \sin ^{2}\left(\theta+\theta_{0}\right)+b^{2} \cos ^{2}\left(\theta+\theta_{0}\right)+c^{2}}}+b \sin \left(\theta+\theta_{0}\right) \\
\frac{a b r_{s} \sin \left(\theta_{s}+\theta_{s o}\right)}{\sqrt{a^{2} \cos ^{2}\left(\theta+\theta_{0}\right)+b^{2} \sin ^{2}\left(\theta+\theta_{0}\right)} \sqrt{a^{2} \sin ^{2}\left(\theta+\theta_{0}\right)+b^{2} \cos ^{2}\left(\theta+\theta_{0}\right)+c^{2}}}+c \theta
\end{array}\right]
$$

Similarly, left to weave strands, wire wound around the left side of the line wires expression vector space quadratic equation formula (7):

$$
P=\left[\begin{array}{c}
\frac{-r_{s} b \cos \left(\theta+\theta_{0}\right) \cos \left(-\theta_{s}+\theta_{s 0}\right)}{\sqrt{a^{2} \cos ^{2}\left(\theta+\theta_{0}\right)+b^{2} \sin ^{2}\left(\theta+\theta_{0}\right)}}+\frac{a c r_{s} \sin \left(\theta+\theta_{0}\right) \sin \left(-\theta_{s}+\theta_{s o}\right)}{\sqrt{a^{2} \cos ^{2}\left(\theta+\theta_{0}\right)+b^{2} \sin ^{2}\left(\theta+\theta_{0}\right)} \sqrt{a^{2} \sin ^{2}\left(\theta+\theta_{0}\right)+b^{2} \cos ^{2}\left(\theta+\theta_{0}\right)+c^{2}}}+b \sin \left(\theta+\theta_{0}\right) \\
\frac{-r_{s} a \sin \left(\theta+\theta_{0}\right) \cos \left(-\theta_{s}+\theta_{s o}\right)}{\sqrt{a^{2} \cos ^{2}\left(\theta+\theta_{0}\right)+b^{2} \sin ^{2}\left(\theta+\theta_{0}\right)}}-\frac{b \cos \left(\theta+\theta_{0}\right) \sin \left(-\theta_{s}+\theta_{s o}\right)}{\sqrt{a^{2} \cos ^{2}\left(\theta+\theta_{0}\right)+b^{2} \sin ^{2}\left(\theta+\theta_{0}\right)} \sqrt{a^{2} \sin ^{2}\left(\theta+\theta_{0}\right)+b^{2} \cos ^{2}\left(\theta+\theta_{0}\right)+c^{2}}}+a \cos \left(\theta+\theta_{0}\right) \\
\frac{-a b r_{s} \sin ^{2}\left(-\theta_{s}+\theta_{s 0}\right)}{\sqrt{a^{2} \cos ^{2}\left(\theta+\theta_{0}\right)+b^{2} \sin ^{2}\left(\theta+\theta_{0}\right)} \sqrt{a^{2} \sin ^{2}\left(\theta+\theta_{0}\right)+b^{2} \cos ^{2}\left(\theta+\theta_{0}\right)+c^{2}}}+c \theta
\end{array}\right]
$$

\section{Geometric Model of Anti-twist Braided Wire Rope.}

Geometric Model. According to the spatial curve equation of strands, draw the centerline of each wire with the three-dimensional modeling software PROE as shown in Fig.3. Finally, sweep along the cross section of the wire rope center line into three-dimensional model as shown in Fig.4.

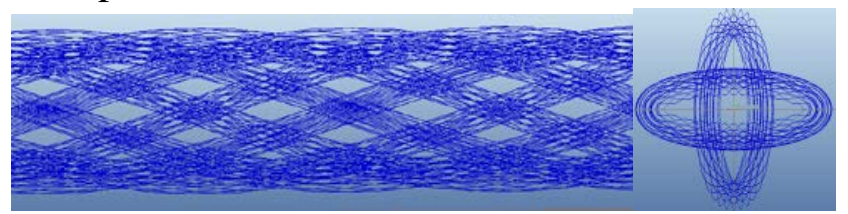

Fig. 3 Centerline of YS9-8×19 braided wire rope geometric model

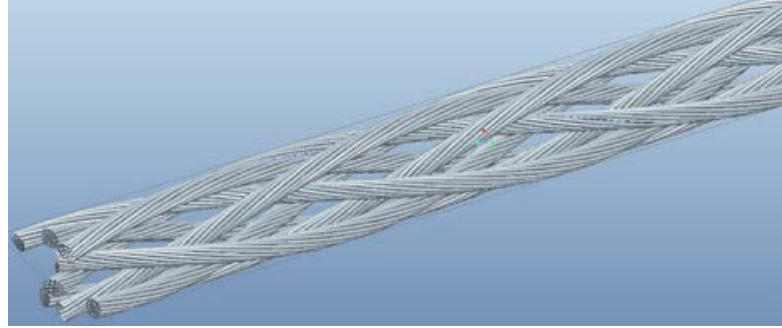

Fig. 4 YS9-8×19 braided wire rope geometric model

Analysis and Comparison of Model. The structural parameters of geometry and standard anti-twisting braided rope were compared, and the results are shown in Table 1.

Table 1 Comparison results of geometric model to standard

$$
\text { Nominal diameter side/mm Shares diameter } / \mathrm{mm} \quad \mathrm{Pitch} / \mathrm{mm}
$$

\begin{tabular}{cccc} 
Model parameters & 11.8 & 2.6 & 126 \\
Standard parameters & 11 & 2.5 & 132 \\
Standard deviation & $\pm 10 \%$ & $0 \sim+8 \%$ & $\pm 10 \%$ \\
Actual deviation & $+7.3 \%$ & $+0.4 \%$ & $-4.5 \%$ \\
\hline
\end{tabular}


As can be seen from Table 1, the geometric model with anti-twist rope braided standard error of three basic structural parameters are within the allowable deviation range. Therefore, the geometric model parameters and standard anti-twist rope consistent, reasonable and feasible mathematical model shows that established in this study.

\section{Conclusion}

(1) According to the forming principle of anti-twist wire rope, the structure and dimensional characteristics of wire rope are analyzed. Applying the differential geometry theory and principle of coordinate transformation, the spatial curve parametric equations were derived. Deduced the mathematical model of anti-twist braided wire rope.

(2) On the basis of this mathematical model ,a YS11-8×19 wire rope was taken as an example based on the spatial curve parametric equations, an geometric entity model of the anti-twist braided wire rope was established using the three-dimension software.

(3) Compared geometric model with actual anti-twist braided wire rope, errors of structure parameters are in the range of allowable error. The mathematic model's veracity is verified,which lay the foundation for finite element analysis of wire rope.

\section{References}

[1]Giglio M, Manes A. life prediction of a wire rope subjected to axial and bending loads[J].Engineering Failure Analysis, 2005, 12(4): 549-568.

[2]Jiang W G. A concise finite element model for pure bending analysis of simple wire strand[J]. Int.J.Mech.Sci.,2012,54:69-73.

[3]Cengiz Erdonmez,C Erdem Imrak.A finite element model for independent wire rope corewith double helical geometry subjected to axial loads[J].lndian Academy of Sciences,2011,36(6): :995-1008.

[4]H Usabiaga, J M Pagalday. Analytical procedure for modelling recursively and wire by wire stranded ropes subjected to traction and torsion loads[J]. International Journal of Solids and Structures. 2008, 45(21): 5503-5520.

[5]Wang D G,Zhang D K,Zhang Z F,etal.Effect of various kinematic parameters of mine hoist on fretting parameters of hoisting rope and a new fretting fatigue test apparatus of steel wire[J].Eng.Fail. Anal.,2012,22:92-112.

[6]Wang D G,Zhang D K, Wang S Q, etal.A finite element analysis of hoisting rope and fretting wear evolution and fatigue life estimation of steel wires[J]. Eng.Fail. Anal.,2013,27:173-193. 